\title{
Noise Reduction of Communication Signal by utilizing the Adaptive Fuzzy-Fractal Filtering Method Cheng Shuang-shuang ${ }^{1,2, a^{*}}$, Li Bing ${ }^{1,2}$, Dong Jun ${ }^{1}$ and Han Hui ${ }^{1}$ \\ ${ }^{1}$ The State Key Laboratory of Complex Electromagnetic Environment Effects on Electronics and Information System (CEMEE), Luoyang, 471003, China \\ ${ }^{2}$ Forth Department Mechanical Engineering College, Shijiazhuang, 050003, China \\ ashuanga0102@163.com
}

Keywords: communication signal; fractal dimension; adaptive fuzzy-fractal filter; fuzzy control parameter.

Abstract. In this paper an adaptive fuzzy-fractal filtering method (AFFF) has been applied to noise reduction of communication signal. Short-time fractal dimension contains the information related to the complexity or characteristic of the time series and it can be used to adjust the value of fuzzy control parameter for improving the filtering effect. Four types of communication signals, including 2ASK signal, 2FSK signal, 2PSK signal and 16QAM signal, are employed to verify the feasibility of the AFFF. Simulation results reveal that the AFFF is superior to the traditional Kalman filter in noise reduction of communication signal. Meanwhile, the AFFF requires low computation cost and is very suitable for noise reduction of communication signal.

\section{Introduction}

The purpose of communication is to achieve the transmission of information via electromagnetic waves [1]. However, the impulse response of the transmission channel with additive external noise makes the quality of received communication signal degradation. The signal restoration system estimates the input signal by both removing the distortion due to the transmission path and also eliminating the corruption of noise [2].

In order to efficiently demodulate the valid signal in the noise environment, a valid filtering technology is needed. The concept of the fractal dimension can be used to measure the complexity of the time series. For different signals, their values of fractal dimension are unequal. In this case, a times series can be classified by using the numeric value of the fractal dimension. This is why the fractal dimension can be applied to signal recognition and signal detection. To data, filtering methods based different types of fractal dimension such as short-time fractal dimension, boxcounting dimension and so on has been proposed and widely applied to engineering problems especially digital signal processing (DSP) problems. For example, an adaptive fuzzy-fractal approach based short-time fractal dimension has been applied to fault diagnosis of rolling bearing in armored vehicle [4], gravitational signal processing [5] and railway signal processing [6]. A fuzzyfractal approach for plant monitoring and diagnostics [7] and forecasting financial and economic time series [8] based box-counting dimension was reported. However, the application of the filtering methods based fractal dimension to communication signal processing remains poorly understood. In this paper an adaptive fuzzy-fractal approach is used to process the communication signal and compares the filtering effect with traditional filtering method such as the Kalman filter.

\section{Fractal Dimension}

Box-counting Fractal Dimension. Box-counting fractal dimension is a simple form of fractal dimension. Grid in $R^{n}$ is obtained by dividing $R^{n}$ into the smaller boxes. For example, there are square grid, rectangle grid and triangle grid in $R^{2}$. X is a compact set of $R^{n} . R^{n}$ is divided into a square grid as small size as possible, and the $X$ is dispersed into a digital collection at the same time. The $N(r)$ is points of $\mathrm{X}$ in discrete space while the size of grid is $r$, i.e., the width of square 
box in grid is $r$. Similarly, the $N(k r)$ is points number of $X$ while the size of box is $k r$. $k$ Points with different sizes of box will be given as $N(k r), k=1,2, \ldots, K$. Where $\mathrm{K}$ is large enough so that $N(\mathrm{kr})>1$.

Suppose

$$
\begin{aligned}
& \mathrm{x}_{\mathrm{k}}=\ln \mathrm{k} \\
& \stackrel{1}{\mathrm{f}} \mathrm{y}_{\mathrm{k}}=\ln \mathrm{N}(\mathrm{kr})
\end{aligned}, \mathrm{k}=1,2, \ldots, \mathrm{K}
$$

The box-counting fractal dimension (for particular size square box is $r$ ) of the set $\mathrm{X}$ can be defined based with the following equation [7]:

$$
\ln N(r)=\ln C-D_{b} \ln r
$$

Here $\mathrm{N}(\mathrm{r})$ is the number of boxes covering $\mathrm{X}$ in grid, $\mathrm{r}$ is the size of box, $\mathrm{C}$ is a constant and $\mathrm{D}_{\mathrm{b}}$ is the box-counting fractal dimension. The least squares fitting of the box-counting fractal dimension can be derived as following equation by (1) and (2).

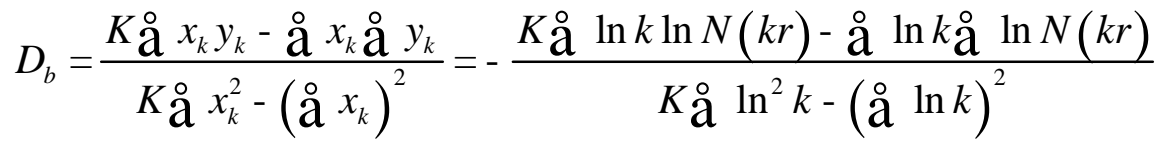

When the resolution $r$ is known, the approximate results

of $D_{b}$ is expressed as follows:

$$
\mathrm{D}_{\mathrm{b}} \gg \ln \mathrm{N}(\mathrm{r}) / \ln \mathrm{K}
$$

Here $K=\max \{k|N(k r)\rangle 1\}$, $k r$ is the width of square box covering $X$.

Short-time Fractal Dimension. Short-time fractal dimension is an algorithm based on boxcounting fractal dimension. For a signal $S$, at a certain time point $t_{k}$, its short-time fractal dimension is defined as the box-counting fractal dimension of the time period $\left[t_{k}-\mathrm{D}, t_{k}\right]$. In the time period $\left[t_{k}-\mathrm{D}, t_{k}\right]$ of time point $t_{k}$, the signal comprises $\mathrm{n}$ ( $\mathrm{n}$ is an even number) sampling points: $\left(\mathrm{x}_{1}^{\mathrm{k}}, \mathrm{x}_{2}^{\mathrm{k}}, \ldots, \mathrm{x}_{\mathrm{n}}^{\mathrm{k}}\right)$.

$$
\begin{aligned}
& D^{k}(r)=\stackrel{\circ}{a}\left|x_{j}-x_{j+1}\right| \\
& D^{k}(2 r)=\stackrel{\mathrm{a}}{\mathrm{a}} / 2_{\mathrm{j}=1}^{\mathrm{o}}\left(\max \left\{\mathrm{x}_{2 \mathrm{j}-1}, \mathrm{x}_{2 \mathrm{j}}, \mathrm{x}_{2 \mathrm{j}+1}\right\}-\min \left\{\mathrm{x}_{2 \mathrm{j}-1}, \mathrm{x}_{2 \mathrm{j}}, \mathrm{x}_{2 \mathrm{j}+1}\right\}\right) \\
& \mathrm{N}^{\mathrm{k}}(\mathrm{r})=\mathrm{D}^{\mathrm{k}}(\mathrm{r}) / \mathrm{r} \\
& \mathrm{N}^{\mathrm{k}}(2 \mathrm{r})=\mathrm{D}^{\mathrm{k}}(2 \mathrm{r}) / 2 \mathrm{r}
\end{aligned}
$$


Where $\mathrm{N}^{\mathrm{k}}(\mathrm{r})$ and $\mathrm{N}^{\mathrm{k}}(2 \mathrm{r})$ respectively is the number of lattices covered signal segment $\left[t_{k}-\mathrm{D}, t_{k}\right]$ while the size of square grid is $\mathrm{r}$ and $2 \mathrm{r}$. Counting the short-time fractal dimension at the time point $t_{k}$ according to (4), we can deduce the following equation:

$$
d_{N}^{k}=\left(\ln N^{k}(r)-\ln N^{k}(2 r)\right) / \ln 2
$$

\section{Adaptive Fuzzy-Fractal Filter}

The mathematical expression of a first-order auto-regressive smoothing filter has been put forward as follows [3]:

$$
\mathrm{y}(\mathrm{i})=a \mathrm{x}(\mathrm{i})+(1-a) \mathrm{y}(\mathrm{i}-1) \quad 0<a £ 1
$$

Where $\mathrm{x}(\mathrm{i})$ is the filter input signal, $\mathrm{y}(\mathrm{i})$ is the output signal after filtering and $a$ is the fuzzy control parameter in the interval $(0,1)$. The simulation results in [3] show that the filter designed by (7) cannot work effectively when the value of parameter $a$ is too large or too small. When $a$ is too large, the signal after filtering will mix lot of noise so that has an impact on post-processing. When $a$ is too small, filtering excesses will be happened and the valid signal received will have a great loss despite the smoothing effect is strengthened. Hence, compared to a fixed value, a parameter $a$ with adaptive capacity is proved to enhance the effectiveness and quality of the filtering process. The adaptation of the filter based (7) depends on the adaptation of parameter $a$. In the filtering process, parameter $a$ should be made dynamic adjustments based the noise level of the input signal and the smaller value corresponds to the stronger noise signal.

Short-time fractal dimension measures dynamically the volatility of signal in different time periods by the definition of short time fractal dimension. This conforms the adaptive require of the parameter $a$ and provides $a$ with a better way to adjust value dynamically with the level of noise signal. On the basis of the above consideration, the approach using the short-time fractal dimension of the signal as a feature to control the parameter a making the filter based the (7) adaptive is proposed.

Suppose parameter $a$ is function of the short-time fractal dimension, i.e. $a=\mathrm{f}\left(\mathrm{d}_{\mathrm{N}}\right)$. Similarly, in different time periods of the input signal, parameter $a$ should be changed with the change of short-time fractal dimension obeying the function $a^{\mathrm{k}}=\mathrm{f}\left(\mathrm{d}_{\mathrm{N}}^{\mathrm{k}}\right)$. The mapping of function $\mathrm{f}$ can be designed according to different needs of different signals. The general guidelines are that, for stronger noise, the waveform of signal is more tortuous, $\mathrm{d}_{\mathrm{N}}^{\mathrm{k}}$ is correspondingly larger and $a^{\mathrm{k}}$ shall be taken as a small value. For weaker noise, waveform of signal is relatively flat, $d_{N}^{k}$ is correspondingly smaller and $a^{\mathrm{k}}$ shall be taken as a lager value. In the simulation of this article, the function is taken as the following piece-wise linear relationship [5]:

$$
\begin{aligned}
& \text { ̀े } a^{k}=-(4 / 7) d_{N}^{k}+1 \quad\left(1<d_{N}^{k}<1.5\right) \\
& \text { 角 } a^{k}=-(1 / 3) d_{N}^{k}+2 / 3 \quad\left(1.5<d_{N}^{k}<2\right)
\end{aligned}
$$

\section{The Simulation Result}

There are a variety of communication signal modulation schemes such as amplitude shift keying (ASK), frequency shift keying (FSK), phase shift keying (PSK) and quadrature amplitude modulation (QAM), etc. For ASK, FSK and PSK, binary modulation denoted as 2ASK, 2FSK and 2PSK is the most basic. In addition there are multi-band modulation such as MASK, MFSK, MPSK and MQAM (M is integer power of 2). 
In this paper we select communication signals 2ASK, 2FSK, 2PSKand 16QAM polluted by white noise as the received signals. The signal sampling frequency is $10000 \mathrm{~Hz}$. Part of the signal within $2 \mathrm{~s}$ was taken to analysis (the figures is given within $0.1 \mathrm{~s}$ ) and the standard differential of white noise is 0.4 . Each 64 sampling points of the received signal is divided into a frame (the time period in section II) to calculate the short-time fractal dimension obeying (5) and (6). The received signals are processed by adaptive fuzzy-fractal filter and Kalman filter separately. The simulation results are showed as Fig.1, Fig.2 and Fig.3.

In order to compare more directly the filtering performance of two types of filtering method, the SNR of the unprocessed signal or the signal processed by adaptive fuzzy-fractal filter and Kalman filter is used to evaluating the quality of treatments. Table 1 shows the simulation results of four types of communication signals. Referring to Table 1, SNR0 is the SNR value of the unprocessed signal, SNR1 is the SNR value of the signal data processed by adaptive fuzzy-fractal filter, SNR2 is the SNR value of the signal processed Kalman filter.

Table 2 shows the simulation results of computing time of four types of communication signal with the AFFF and Kalman filter. The $t_{A}$ is the computing time of the AFFF and the $t_{K}$ is the computing time of Kalman filter.
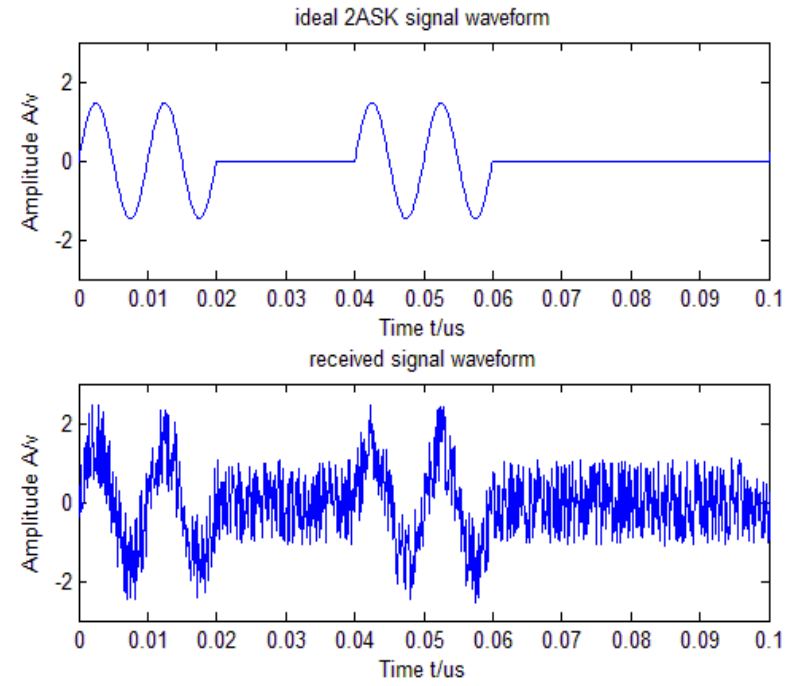

Fig. 1 Ideal 2ASK signal waveform and received signal waveform

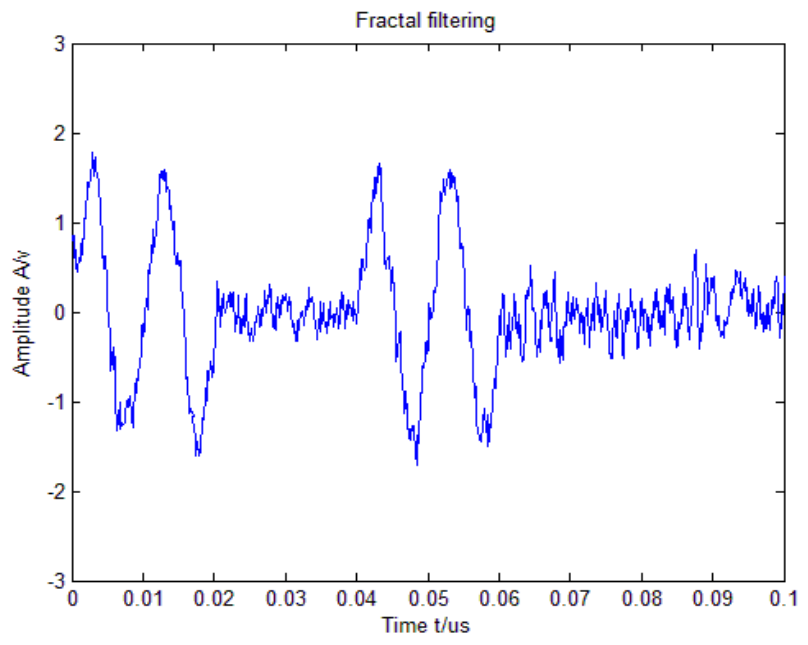

Fig. 2 Processed waveform by fractal filter 


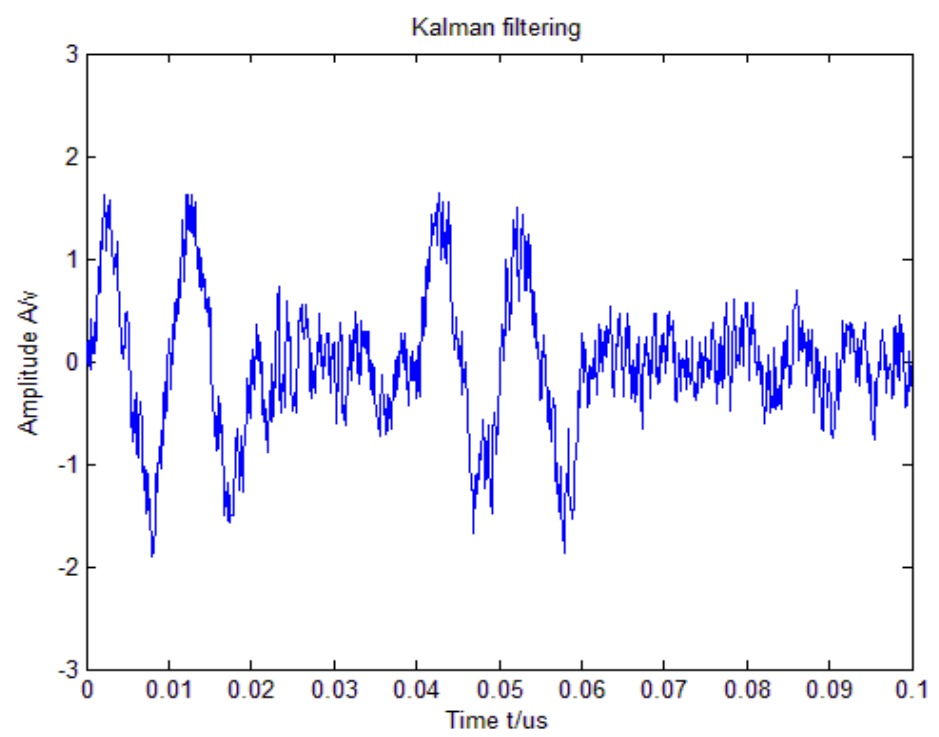

Fig. 3 Processed waveform by kalman filter

Table. 1 Comparison of SNR values of four types of communication signal

\begin{tabular}{|c|c|c|c|}
\hline & SNRO & SNR1 & SNR2 \\
\hline 2ASK & 0.9687 & 8.7202 & 6.7429 \\
\hline 2FSK & 1.0189 & 8.2539 & 7.1822 \\
\hline 2PSK & 0.9283 & 7.4929 & 6.8027 \\
\hline $\mathbf{1 6} \boldsymbol{Q A M}$ & 0.9750 & 7.7824 & 7.0331 \\
\hline
\end{tabular}

Table. 2 Comparison of computing time of the AFFF and kalman filter

\begin{tabular}{|c|c|c|}
\hline & $\boldsymbol{t}_{\boldsymbol{A}}(\mathrm{s})$ & $\boldsymbol{t}_{\boldsymbol{K}}(\mathrm{s})$ \\
\hline $\mathbf{2 A S K}$ & 0.0160 & 1.0378 \\
\hline $\mathbf{2 F S K}$ & 0.0162 & 1.0216 \\
\hline $\mathbf{2 P S K}$ & 0.0177 & 1.0226 \\
\hline $\mathbf{I 6 Q A M}$ & 0.0170 & 1.0181 \\
\hline
\end{tabular}

As can be seen from Fig.1, Fig.2 and Fig.3, the adaptive fuzzy-fractal filter and the Kalman filter can suppress the impact of noise on communication signal in a certain extent. Table 1 shows that, for four kinds of the communication signals, the values of SNR1 is smaller than the values of corresponding SNR2, which indicates that the filtering performance of the adaptive fuzzy-fractal filter proposed in this paper is better than Kalman filter. Table 2 shows that, for four kinds of the communication signals, the values of $t_{A}$ is smaller than the values of corresponding $t_{K}$, which indicates the computation efficiency of the AFFF is higher than Kalman filter.

\section{Conclusion}

This investigation has applied an adaptive fuzzy-fractal filtering method (AFFF), which is on the basis of fractal theory and first-order auto-regressive smoothing filter, to noise reduction of communication signal. The AFFF method mainly consists of three factors: short-time fractal dimension, first-order auto-regressive smoothing filter and the function between short-time fractal dimension and fuzzy control parameter. We have evaluated the filtering effect of AFFF method on four types of communication signals and taken the traditional Kalman filter as a comparison. Experimental results have revealed that the AFFF method achieved better performance on noise reduction. Furthermore, the computation efficiency of the AFFF is proved to be very high. 
Therefore, the AFFF method is very suitable for noise reduction of communication signal and it also can be used for noise reduction of other time series.

A future direction of study is to improve the function between short-time fractal dimension and fuzzy control parameter. Another direction is to apply the AFFF to other types of time series.

\section{Acknowledgment}

This research is supported by the State Key Laboratory of Complex Electromagnetic Environment Effects on Electronics and Information System, under project number CEMEE2015K0102B and the National Natural Science Foundation of China, under project number 51205405.

\section{References}

[1] LI Chao-wei, ZHOU Xi-yuan, LIU Fu-lai. Integrative Reconnaissance Technology for Radar and Communication Signals, Shipboard electronic Countermeasure. 31-2 (2008) 5-11.

[2] Bor-Sen Chen, Chin-Wei Lin. Multiscale Wiener Filter for the Restoration of Fractal Signal: Wavelet Filter Bank Approach, IEEE Transaction on Signal Processing. $42-11$ (1994) 2972-2982.

[3] ZHU Rong-fu, YE Nian-yu. A Fuzzy Automatic Control Fractal Filter, J. Huazhong Univ. of Sci. \& Tech. 29-12 (2001) 61-63.

[4] YAO Zhu-ting,, PAN Hong-xia, CHEN Yue-lian. Application of Fractal Theory in Fault Diagnosis of Armored Vehicle Rolling Bearing, Journal of Gun Launch \& Control. 4 (2009) 66-70.

[5] ZHAO Li-ye, ZHOU Bai-ling, ZHAO Chi-hang, MA Yun-feng. Application of Fractal Filtering in Gravitational Signal Processing, Journal of Chinese Inertial Technology. 29-12 (2004) 32-36.

[6] MA Xue-jie, WEI Xue-ye. Fractal Filtering and Its Application in Railway signal, Journal of Traffic and Transportation Engineering. 1-1 (2001) 58-60.

[7] Oscar Castillo, Patricia Melin. A New Hybrid Approach for Plant Monitoring and Diagnostics Combining Type-2 Fuzzy Logic and Fractal Theory, IEEE. 7461 (2001) 111-116.

[8] Oscar Castillo, Patricia Melin. A New Fuzzy-Fractal Approach for Forecasting Financial and Economic Time Series, IEEE. 7078 (2001) 929-934.

[9] Xu Guang. Vibration Analysis and Fault Diagnosis for Fault Gera(Master Dissertation), Yanshan University, 2012. 